\title{
El estándar DCI en las filmotecas. El proceso de transición al sistema digital en la actividad filmotecaria contemporánea (2010-2014)
}

\author{
Pablo García-Casado*, Jordi Alberich-Pascual** \\ * Director de la Filmoteca de Andalucía. Correo-e: pablo.garcia@juntadeandalucia.es \\ ** Facultad de Comunicación y Documentación de la Universidad de Granada. Correo-e: jalberich@ugr.es
}

Recibido: 25-11-2014; 2a versión: 31-03-2015; Aceptado: 20-04-2015.

Cómo citar este artículo/Citation: García-Casado, P.; Alberich-Pascual, J. (2015). El estándar DCI en las filmotecas. El proceso de transición al sistema digital en la actividad filmotecaria contemporánea (2010-2014). Revista Española de Documentación Científica, 38(4): e107. doi: http://dx.doi.org/10.3989/redc.2015.4.1241

\begin{abstract}
Resumen: El presente artículo expone el estado actual e implicaciones del cambio hacia el nuevo paradigma digital en el sector cinematográfico y en las filmotecas con motivo de la implantación del sistema DCI (Digital Cinema Initiatives) como estándar internacional de producción, distribución y exhibición cinematográfica. Tras analizar los paralelismos existentes entre este acuerdo de normalización, impulsado por los principales estudios cinematográficos en 2002, y los acuerdos tomados por esas mismas empresas casi cien años antes a mediados de la segunda década del siglo pasado, se debaten finalmente los efectos y consecuencias para la actividad filmotecaria contemporánea en su transición al sistema digital DCI como nuevo estándar de exhibición y conservación fílmica.
\end{abstract}

Palabras clave: DCI; cine; filmoteca; digitalización; estandarización; exhibición; conservación.

The DCI standard in film archives. The transition to digital systems in the contemporary activity of film archives (2010-2014)

\begin{abstract}
This article sets out the current state of the shift towards a new digital paradigm in the world of cinema with the implementation of the DCI system (Digital Cinema Initiatives) as the international standard of cinema production, distribution and exhibition. The article also analyses the parallels between this agreement for standardization, driven by the major film studios in 2002, and the agreements made by these same companies almost a hundred years earlier, in the middle of the second decade of the last century. In addition, the article presents how film archives are experiencing this digital transition and how they have accepted the normalization offered by the DCI as the standard for film conservation and exhibition.
\end{abstract}

Keywords: DCI; cinema; film archive; digitalization; standardization; exhibition; conservation.

Copyright: (c) 2015 CSIC. Este es un artículo de acceso abierto distribuido bajo los términos de la licencia Creative Commons Attribution-Non Commercial (by-nc) Spain 3.0. 


\section{INTRODUCCIÓN}

El cine es tecnología. Es hijo de la revolución industrial, un invento datado por el hombre, y en el que cada momento tecnológico destacado tras su invención ha afectado decisivamente la manera de producir, distribuir y exhibir las películas. La llegada del sonoro, el acceso al color, la aparición de la televisión y del vídeo o la emergencia de los nuevos soportes digitales han sido cuestiones sustanciales, no accesorias, que han cambiado el paradigma, la manera de hacer y de ver cine, y que han tenido un impacto decisivo en la configuración y desarrollo histórico del propio discurso cinematográfico (Bordwell, 1979; Gubern, 1987; Wyver, 1989; Miller y Stam, 1999). En el reciente cambio de siglo, numerosos autores aventuraron ya como la llegada del nuevo paradigma digital pondría en cuestión las bases tradicionales sobre las que se había fundado la industria cinematográfica, tanto en el proceso de producción, como en las fases de distribución y exhibición (Cubitt, 1998; Manovich, 2001; Darley, 2002; Thorburn y Jenkins, 2004).

El presente artículo tiene como objetivo específico analizar el alcance de tal cuestionamiento en el marco de la adopción reciente del nuevo estándar digital DCP/DCI por parte de las filmotecas contemporáneas, una vez superado el debate en el seno de la propia Federación Internacional de Archivos Fílmicos sobre si resultaba filmotecariamente válida o no la proyección en formatos digitales (Francis, 2002; García-Casado y Alberich-Pascual, 2014). Nacidas en el contexto de la tercera década del siglo pasado como instituciones destinadas a la conservación de un patrimonio (fílmico) físico y material, y a la defensa de la cultura cinematográfica, el nuevo escenario digital ha transformado de forma radical el quehacer diario de las filmotecas (Schaefer y Streible, 2007). La transición tecnológica digital, no es ya una incierta expectativa de futuro para la actividad filmotecaria, sino su presente y futuro cierto. La identidad e incluso la supervivencia misma de las filmotecas está en juego en su adaptación o no a las nuevas tecnologías de naturaleza digital (Meyer, 2005; Fossati, 2009).

\section{ASPECTOS METODOLÓgICOS}

El planteamiento discursivo de este trabajo se fundamenta en la adopción creciente e históricamente revolucionaria en el último lustro (20102014) de los nuevos estándares digitales DCDM/ DCI y DCP/DCI por parte de las filmotecas contemporáneas para su labor de conservación y exhibición fílmica digital. En este trabajo revisamos este cambio y sus consecuencias para la actividad filmotecaria. Para ello, exploramos los paralelismos y similitudes del proceso de implantación del estándar de 35 milímetros, que permitió la consolidación de la industria cinematográfica moderna (Musser, 1991), y que ha sido el estándar para la conservación y exhibición filmotecaria desde la fundación de estas instituciones hasta la actualidad (Borde, 1991; Francis, 2002; Costa, 2004; Del Amo, 2011), en relación con el proceso reciente de implantación del sistema digital DCI a lo largo de la última década, tanto en la industria cinematográfica como en filmotecas.

Nuestra aproximación metodológica está condicionada por la escasez de material bibliográfico y de literatura científica de impacto sobre el tema. Las referencias en bases de datos sobre el proceso de digitalización de filmotecas y archivos fílmicos tienden sobre todo a la especialización, a casos muy concretos de restauraciones y de análisis retrospectivos de cinematografías, pero son pocas las muestras de una bibliografía adecuada sobre el papel que comporta la adopción de un determinado estándar de calidad cinematográfica digital para la definición, identidad y actividad de las propias filmotecas, así como sobre el sistema DCI que nos ocupa. Procede igualmente la cautela, pues constatamos como los diagnósticos recientes sobre la implantación de la tecnología digital en el sector cinematográfico realizados hace menos de un lustro, han quedado o quedarán en breve superados por la realidad (Izquierdo, 2007; Paz-García, 2010; García-Santamaría, 2013).

El presente estudio ha conjugado investigación básica y aplicada, descriptiva y de campo. Hemos acudido a publicaciones técnicas aplicadas, especialmente las existentes en los estudios parciales que sobre el tema viene realizando la Federación Internacional de Archivos Fílmicos. En el Anexo 1 consignamos el listado de filmotecas pertenecientes a la FIAF de las que actualmente existen datos sobre la existencia o no de tecnología DCI. Hemos mantenido igualmente entrevistas puntuales con los responsables y jefes de conservación de un elenco de filmotecas nacionales (Filmoteca Española, Filmoteca de Catalunya, Filmoteca de Galicia (CGAI), Filmoteca Valenciana, y Filmoteca de Andalucía) que nos ha permitido recabar ejemplos prácticos de esta transición. Asimismo, se han obtenido y estudiado las fuentes legislativas en relación con la transición digital del sector cinematográfico. Todo ello nos ha permitido elaborar el siguiente análisis crítico sobre la implantación del 
reciente estándar digital DCI en la actividad filmotecaria contemporánea (2010-2014).

\section{EL ESTÁNDAR DCI EN LAS FILMOTECAS}

\subsection{El estándar de $35 \mathrm{~mm}$}

El año 1909 marcó un antes y un después en el devenir de la industrialización e institucionalización de la producción cinematográfica moderna, con la constitución en Estados Unidos de un consorcio de empresas formado para evitar la entrada de patentes extranjeras y también para cerrar el mercado a posibles competidores dentro del país encabezado por Thomas Alva Edison: la Motion Picture Patents Company (Talens y Zunzunegui, 1997; Mezias y Boyle, 2005). La MPPC estableció unas normas específicas oligopolísticas en cuanto a la producción, la gestión, la distribución y la comercialización cinematográfica.

El trust de Edison trató de defender sus intereses proponiendo demandas por infracción de patentes, como una forma de proteger sus invenciones y las ganancias, no sin una cierta aveniencia de Washington, abocada a elegir entre la necesidad de consolidar una industria emergente, algo que le proporcionaba la MPPC, y la necesidad de responder contundentemente a un consorcio que contravenía los valores inspiradores de una economía liberal concretados en la Ley Antimonopolio Sherman (Mezias y Boyle, 2005). Frente a esta actitud oligopolística de la MPPC, emergieron como oposición un grupo de emigrantes europeos que se establecieron por su cuenta en una colina cercana a Los Ángeles Ilamada Hollywood. Esos autollamados "productores independientes" iniciaron una intensa batalla legal, hasta que el 1 de octubre de 1915 la corte federal de Estados Unidos resolvió que las actividades de la MPPC habían ido mucho más allá de lo necesario para proteger el uso de las patentes (Nowell-Smith, 1996; Manley, 2011).

Ese mismo año de 1915, en lo que supondrá un duro golpe para los intereses de la MPPC, Charles Francis Jenkins, el ingeniero que había desarrollado uno de los primeros proyectores de cine en 1895 junto con Thomas Alva Edison, pero que ahora trabajaba para Columbia, una de las competidoras de Edison, inicia los contactos para crear la Motion Pictures Engineers, una entidad a priori instrumental, que reuniese a técnicos y no a productores, propietarios o economistas (Curtis, 2004).
Un año después, en julio de 1916, Jenkins, E. K. Gillett y N. I. Brown, junto con siete ingenieros adicionales se reunieron en Washington DC, donde tomaron la decisión unánime de crear una sociedad de ingenieros especialistas en el campo de la imagen en movimiento: la Sociedad Cinematográfica de Ingenieros (SMPE), siendo nombrado el propio Jenkins primer presidente de la nueva sociedad en su acta de constitución (Musser, 1991). En octubre de ese mismo año, en Nueva York se producirá la segunda reunión de la SMPE, en la que se fijará definitivamente el estándar del $35 \mathrm{~mm}$. a partir del diseño previo del ingeniero Donald J. Bell, y en la que se acordarán igualmente los comités técnicos de cámaras, equipamientos, ópticas y lámparas en relación al nuevo estándar (Jones, 1933).

En este contexto, los productores independientes de Hollywood, aprovechando a) la aplicación de la denominada Doctrina Sherman, b) el acuerdo de resolución de conflictos pactado con la MPPC, y c) los trabajos de la SMPE en cuanto al diseño del estándar del 35 mm., crearon un sistema que actúo de barrera frente a nuevos competidores al cerrar la industria a todo operador económico que no aceptase un tipo estándar de película, lo que aseguraba a su vez una dura restricción tecnológica a todos los niveles de la cadena productiva, desde las cámaras de filmación y los laboratorios, hasta las moviolas y los proyectores.

La estandarización del ancho de 35 milímetros habría de tener un impacto trascendental en el desarrollo posterior tanto de la industria como de la difusión cinematográfica al convertirse de facto en un estándar internacional: "La aceptación inicial del $35 \mathrm{~mm}$. como ancho de vía estándar hizo posible que las películas se mostrasen en todos los países del mundo. Se proporcionó un formato uniforme y fiable y predecible para la producción, distribución y exhibición de películas, lo que facilitó la rápida difusión y aceptación de las películas en todo el mundo como dispositivo central para el entretenimiento y la comunicación" (Fullerton y SöderberghWidding, 2000).

Pese a la posterior irrupción de la televisión, o al cambio de paradigma que supuso la entrada de la tecnología vídeo, y pese a los diversos augurios sobre la muerte del cine (Cherchi Usai, 1995), el estándar 35 milímetros, con distintas variaciones, incorporando sonido, color, modificando la emulsión, modificando su relación o ampliando sus posibilidades lumínicas, habría de resistir hasta la actualidad como estándar indiscutible para la industria ciematográfica internacional, así como 
estándar y seña de identidad para la propia conservación y difusión filmotecaria (Francis, 2002; Del-Amo y Fernández, 2011).

\subsection{La emergencia del estándar DCI}

La irrupción y progresiva incorporación del soporte digital en el ámbito cinematográfico a partir de la última década del siglo $X X$ ha resultado una amenaza para la supervivencia del modelo de producción, distribución y exhibición fílmica alrededor del estándar de 35 milímetros (Hanson, 2004). La tecnología digital ha permitido un abaratamiento revolucionario de los costes de producción cinematográfica, así como una accesibilidad masiva a dispositivos de calidad cinematográfica. Este intenso proceso de cambio ha implicado asimismo la apertura del sector y del mercado a nuevos agentes y modelos de negocio ajenos a los hegemónicos durante más de un siglo de la mano del 35 milímetros (Hartley, 2008; Christopherson, 2008).

La optimización en el último lustro de la tecnología de captura y registro audiovisual digital permite ya rodar una película en formato aficionado y distribuirla masivamente. El abaratamiento de los soportes digitales ha hecho que el coste de revelado y copiado de cualquier película en soporte fotoquímico sea significativamente mayor que el coste de cualquier disco duro convencional que puede almacenar hasta 1TB de capacidad. La edición y la posproducción cinematográfica pueden resolverse satisfactoriamente en la actualidad mediante el uso de software libre, y formatos audiovisuales digitales destinados inicialmente a uso doméstico permiten -asociados adecuadamente con otras herramientas no profesionales-, visionar y proyectar películas en alta definición. El escalonamiento habitual del ciclo de vida de una producción cinematográfica ideado en la década de los ochenta, esto es, $1^{\circ}$ ) estreno en salas; $2^{\circ}$ ) difusión en soporte vídeo/cable, $3^{\circ}$ ) televisión generalista, entrará en crisis a partir del cambio de siglo con la profusión creciente de las más diversas y heterogéneas pantallas, plataformas y vías de acceso digital a las obras cinematográficas (Tubella y otros, 2008).

El dominio del sistema digital en el conjunto del proceso de producción cinematográfica, desde su fase inicial de preproducción, hasta alcanzar su postproducción, arte final y exhibición pública ha obligado a redefinir en unos pocos años el conjunto de la industria audiovisual nacional e internacional desde un modelo precedente de naturaleza física y material, a un nuevo modelo de naturaleza electrónica e inmaterial (García-Santamaría, 2013).
El fin del estándar del 35 milímetros, no por anunciado y previsto, ha resultado menos abrupto. Las empresas que fabricaban industrialmente el propio soporte fotoquímico cinematográfico tomaron ya la decisión de dejar de producir material fotoquímico a partir de 2013. La propia Unión Europea, consciente de esta realidad, ha acelerado los programas de ayuda a la digitalización de salas privadas, abocando a aquellas empresas que no aborden ese cambio tecnológico a la práctica desaparición por inanición. En Andalucía (España), por ejemplo, estas ayudas que cuentan con la financiación de los fondos FEDER de la UE, se concretaron en la Orden de 8 de agosto de 2013, por la que se establecen las bases reguladoras para concesión de subvenciones, en régimen de concurrencia competitiva, de apoyo a empresas culturales y creativas andaluzas para el fomento de su competitividad, modernización e internacionalización (BOJA 163, 2013: 5).

El fin de facto del estándar de 35 milímetros, así como la expectativa de apertura incontrolada del proceso de distribución y exhibición cinematográfica en salas, empuja a la industria cinematográfica contemporánea a la necesidad de fijación de un nuevo estándar universal para la proyección cinematográfica digital en salas. Disney, Fox, Paramount, Sony Pictures Entertainment, Universal y Warner Bros. Studios, reconvertidas y/o integradas todas ellas desde finales del siglo $X X$ en el seno de unas pocas corporaciones multinacionales de medios, desarrollaron conjuntamente a partir del año 2002 bajo el paraguas de las Digital Cinema Initiatives (DCI) un conjunto coordinado de especificaciones técnicas compartidas para el cine digital industrial que asegurara un nivel uniforme y elevado de prestaciones, fiabilidad y control de calidad.

A través de los acuerdos DCI, las grandes majors cinematográficas han establecido a lo largo de la última década unos parámetros de homologación en cuanto a la producción, la distribución y la exhibición cinematográfica digital: sólo unos modelos de proyectores integrados; sólo un tipo de formato de exhibición; sólo un soporte, marcando así distancias entre lo profesional y lo aficionado, entre la industria y el voluntarismo cultural. En un primer nivel quedan englobadas las salas tradicionales de exhibición cinematográfica y las producciones de alto coste; en un segundo nivel, el mundo del documental, los cines periféricos, los Creative Commons, los cortos, y el conjunto del cine amateur, todos ellos al margen de los nuevos estándares propuestos por el sistema DCI. La industria cinematográfica consorciada persigue así a través 
del nuevo estándar DCI perseverar en lo que el 35 milímetros triunfase durante más de 100 años: a) la limitación de acceso a competidores, b) la instauración de barreras tecnológicas, y c) la uniformidad, fiabilidad y predectibilidad en la calidad de distribución y exhibición cinematográfica.

En las primeras décadas del siglo $\mathrm{XX}$, la industria norteamericana consiguió poblar el planeta de proyectores de 35 milímetros; cien años después se repite la estrategia. Hoy por hoy, la práctica totalidad de los exhibidores españoles poseen ya proyectores bajo el paraguas DCI; igualmente, la práctica totalidad de los productores y distribuidores de cine profesional para exhibición lo hacen en formato DCP, bajo patente DCI. De no hacerlo así, difícilmente podrían ser comercializados puesto que la homologación DCI está reservada a un grupo de marcas escogidas y concretas. Las decisiones de las grandes productoras de Hollywood han arrastrado también al estándar DCI al mercado de cine independiente y a las filmografías de países emergentes. El centro ha arrastrado de nuevo a la periferia, en un proceso de resultados semejantes a los diseñados 100 años atrás para el 35 milímetros.

El éxito reciente del sistema DCI ha creado un estándar que asegura a corto y medio plazo a la industria cinematográfica un control sobre cada una de las fases de la producción, la distribución y la exhibición cinematográficas en el entorno profesional, desplazando al resto de las modalidades del audiovisual digital (no comercial, familiar o amateur) a entornos menos costosos.

\subsection{La adopción filmotecaria del estándar DCI}

Las filmotecas, que nacieron en los años 30 del siglo pasado para preservar el patrimonio fílmico frente al poder del derechohabiente, como vector de resistencia frente a la vocación destructiva que tanto la industria de la producción como la de exhibición y de distribución habían tenido históricamente para con aquellos soportes fílmicos que habían agotado el ciclo de vida económico, no entraron en el debate de la estandarización del 35 milímetros: era ya una cuestión asumida en el sector cinematográfico (Borde y Buache, 1997), lo que no quiere decir que no admitieran otros soportes. De hecho, en la reconstrucción de los primeros años del cine, las filmotecas siguen hoy día rescatando joyas en $16 \mathrm{~mm} ., 9,5 \mathrm{~mm} .$, u $8 \mathrm{~mm}$., entre otros formatos posibles, además de aceptar otros formatos más raros y los posteriores soportes magnéticos. Pero la asunción del estándar de $35 \mathrm{~mm}$. ha dominado enteramente la labor filmotecaria moderna hasta muy recientemente, como lo demuestra que las restauraciones, al menos hasta la primera década del siglo XXI, se realizaran de forma exclusiva en soporte negativo de $35 \mathrm{~mm}$. para la conservación y también de $35 \mathrm{~mm}$. para el tiraje de copias destinadas a la exhibición en filmotecas.

Hasta 2005, la tecnología digital se adopta en las filmotecas de manera tímida y lenta, considerado como un soporte de menor entidad, subsidiario en relación al aún hegemónico $35 \mathrm{~mm}$. Fruto de la enorme desconfianza inicial de las filmotecas hacia lo digital e Internet, aún en 2008, "las filmotecas presentes en internet disponen, en general, de muy pocos contenidos, y en su mayor parte son meramente indicativos de lo que es la institución y de los servicios que presta" (López Yepes, 2008). Pese a su desconfianza inicial para con la tecnología digital, las filmotecas se vieron impelidas a encarar y resolver sus dudas en relación con la conservación, catalogación y exhibición filmotecaria de los soportes digitales, como una realidad industrial, cultural y de mercado de la que no podían, ni debían, sustraerse.

Entre los años 2005 y 2010, la Federación Internacional de Archivos Fílmicos (FIAF), entró en el debate teórico sobre la aceptabilidad o no de éstos en el campo filmotecario, significándose tres enfoques contrapuestos. El primero se corresponde con la resistencia total a estos soportes, encarnado por el portugués José Manuel Costa, de la Cinemateca Portuguesa; un segundo enfoque, de aceptación plena, defendido por Mark Paul Meyer, del Eye Film Institute de Amsterdam; y una posición más ecléctica y condicionada, encabezada por Nicola Mazzanti (García-Casado y Alberich-Pascual, 2014). Todos estos posicionamientos se quedaron aún en el plano teórico, el de la política cultural, sin alcanzar aspectos ni detalles prácticos.

La posterior implantación abrupta del sistema digital en los últimos años provocó que en España pasara sólo en tres años de un $17 \%$ en 2009 a un $41 \%$ en 2011 (García Santamaría, 2013), y que a partir de esa fecha hasta la actualidad (2014), la proyección en soporte analógico sea puramente residual. Esta situación ya la aventuró la FIAF, como organismo de referencia internacional que aglutina los principales archivos fílmicos del planeta, se viera abocada a concluir en 2010 la aceptación (aunque con matizaciones) del estándar DCI para la conservación fílmica digital. 
"Recommendation on the deposit and acquisition of D-cinema elements for long term preservation and access" (FIAF Commissions, 2010), el documento que tras sucesivas reuniones técnicas sectoriales recoje su dictamen, centra su atención en tres tipos de soportes digitales cinematográficos industriales: (1) el Digital Source Master (DSM), el máster digital de producción de la película, que no es en sí mismo la obra que se proyectará sino un soporte bruto de la película, (2) el Digital Cinema Distribution Master (DCDM), el conjunto de los archivos originales para producir todas las copias de proyección en $D$-Cinema, comparable a un negativo fotoquímico. No está estandarizado por lo que puede haber multiplicidad de formatos y soportes, y (3) el Digital Cinema Package (DCP), la "copia digital" que se envía y proyecta en las salas de cine, la obra cinematográfica tal como es vista por el público, estandarizado para permitir una distribución homogénea y efectiva, y que puede estar encriptado o no. De las tres opciones, la FIAF concluirá que "un DCDM o un DCP sin encriptar sí representan formatos aceptables para preservación a largo plazo de una obra cinematográfica", y expone como una opción subsidiaria la aceptación del DSM, "aunque no en lugar de un DCDM o de un DCP" (FIAF Commissions, 2010), señalando asimismo con nitidez la necesidad de la no encriptación como una medida necesaria para el acceso al archivo fílmico: de nada servirá conservar soportes digitales si el acceso a éstos está bloqueado por los productores. De hecho, sería deseable que los protocolos DCI incluyeran en el futuro formatos de código abierto para su depósito en filmotecas y archivos fílmicos (Nowak, 2010).

A partir de ese momento, los formatos DCP/DCI y DCDM/DCI empezarán a ser reconocidos de forma habitual en el panorama filmotecario internacional como sustitutivos y equivalentes digitales del estándar físico de $35 \mathrm{~mm}$. Así, cuando la comisión técnica de la FIAF prepara un cuestionario de preguntas y respuestas acerca de la cuestión de la conservación digital para ilustrar y resolver las dudas que puedan surgir a los asociados de la FIAF, señalará en el documento resultante la idea clara de que la digitalización pasa por el sistema DCI, sin otra alternativa. En ese mismo documento, su coordinador, Torkell Sætervadet de la Filmoteca de Noruega, define ya el formato estándar DCP/DCI de manera clara como "un sistema capaz de producir una imagen de calidad igual a la imagen de la película de 35mm" (Sætervadet, 2006).

Más recientemente, dos personas de referencia en el mundo filmotecario contemporáneo como son el responsable del archivo fílmico sueco, Jon Wengström (2013), y el del BFI británico, Dylan Cave (2013), compartirán y ahondarán en estos mismos planteamientos en sendos artículos publicados en el Journal of Film Preservation: si las obras están en un soporte bajo el estándar DCI, su depósito deberá ser igualmente en el soporte original DCP/DCI. En la actualidad todas las filmotecas admiten ya el depósito de las obras audiovisuales digitales bajo el sistema DCI, aplicando la analogía de conservar las obras en su formato original.

La situación de transición abrupta del estándar $35 \mathrm{~mm}$. a los nuevos estándares digitales queda ejemplificada en el caso español en la diversidad de fórmulas jurídicas existentes aún en la legislación estatal y autonómica para arbitrar el depósito de obra cinematográfica original, y para acceder a las ayudas públicas existentes a la creación y a la producción. Así, mientras el Ministerio de Cultura de España, establece desde 2014 una referencia al depósito de las obras en soporte original en las sucesivas convocatorias de ayudas a la producción cinematográfica, abriendo así con ello la puerta al depósito de obras en formato DCP/DCI (BOE 50, 27 de Febrero de 2014), en normativas como las del País Vasco se mantiene aún una separación entre las obras en soporte fotoquímico y digital (BOPV 107, Orden de 4 de Junio de 2014). Encontramos igualmente normativas no adaptadas, como las de Andalucía, expresada en la Orden de 2011, que regulará las sucesivas convocatorias hasta la actualidad, la necesidad de entregar un "internegativo o en un CRI (color reversible intermedio), para su depósito en la Filmoteca de Andalucía y de la copia obtenida para la entrega obligatoria en dicha Filmoteca" (BOJA 169, 2011).

Salvo aquellos archivos fílmicos que se declaran exclusivamente retrospectivos porque sólo recogen material fotoquímico y/o porque se centran exclusivamente en ser centros de consulta, los reportes anuales recientes de cada una de las filmotecas pertenecientes a la FIAF, en los que cada filmoteca expone el balance de actividades y adquisiciones (Anexo I), constatan que las filmotecas han adoptado muy mayoritariamente el formato DCP/DCI para su actividad de programación y exhibición. A fecha de diciembre de 2013, de los 117 miembros que presentan reportes anuales de actividad a la organización, 77 ya tenían equipos DCP para exhibición y archivo. Entre ellas están las principales filmotecas de referencia en el mundo. Los otros 40 miembros se dividen en los que no acreditan (15) que lo tengan, y los que 
expresan (25) que no poseen esa tecnología, ya sea porque se dedican en exclusiva al material fotoquímico, porque sea una filmoteca que dependa de una matriz que sí la tenga (Filmoteca de Gales) o por meros problemas económicos (Filmoteca de Albania o la Asian Film Archive).

Además del estudio pormenorizado de los datos existentes en la documentación de la FIAF a nivel internacional, procedimos a enviar un cuestionario a un elenco de filmotecas de ámbito nacional (Filmoteca Española, Filmoteca de Catalunya, Filmoteca de Galicia (CGAI), Filmoteca Valenciana, y Filmoteca de Andalucía), que nos ha permitido recabar ejemplos prácticos de cómo han realizado esa transición, así como el nivel de implantación de formatos DCP existente en los fondos de éstas, a partir de las respuestas de Mercedes de la Fuente, directora del Centro de Conservación y Restauración de fondos fílmicos de la Filmoteca Española (Madrid), de Mariona Bruzzo, Jefa del Centro de Conservación y Restauración Filmoteca de Catalunya, de José Ignacio Lahoz, Jefe de Conservación de La Filmoteca-IVAC (Filmoteca Valenciana), de José María Rodríguez Armada, del Archivo CGAI (Filmoteca de Galicia) y de Ramón Benítez, de la Filmoteca de Andalucía.

Todas ellas disponen en la actualidad de sistemas de reproducción para archivos DCP. La Filmoteca Española desde el año 2010; la Filmoteca Valenciana desde 2008; el CGAI desde 2011; y en las Filmotecas de Catalunya y de Andalucía desde 2013. En los casos de Valencia, Catalunya, Andalucía y Galicia, el equipo se utiliza para la exhibición, mientras que para el archivo se utilizan otras herramientas tecnológicas, sea para subir los archivos DCP a un servidor (Catalunya y Andalucía), o para reproducirlas para archivo con software adaptado (Valencia y Galicia). En el caso de la Española, tienen uno en el Cine Doré (Sala de exhibición) y desde 2013 otro en el Centro de Conservación y Restauración, que utilizan también para comprobación de copias, catalogación, etc.

La proporción de copias DCP frente a otros formatos de conservación fílmica en la actualidad es en los cinco casos recabados aún muy baja en relación al conjunto de fondos preexistente fotoquímico, pero todas coinciden en que todas las producciones que reciben subvención entregan ya DCP. En el caso de la Filmoteca de Catalunya, se recibe el primer DCP en 2009; en 2010, 0; en 2011 entran 2; en 2012 entran 12; en 2013, 26; y en 2014,35 . En el caso de la Galicia la recepción de copias en DCP se inicia en 2012, y en An- dalucía en 2011, quedando patente, en todos los casos, que se trata de una variación brusca, pues en tres años han pasado de tener una presencia poco significativa (uno o dos al año) a constituir ya prácticamente el grueso de materiales entregados. Esta realidad se constata en la Filmoteca Española, pues, como señala el organismo, desde el año 2014 las copias DCP representan prácticamente el $100 \%$ de lo producido por la industria del cine. Aún así, sólo hay 150 DCP frente a más de 100.000 materiales fotoquímicos y más de 60.000 en formato electrónico y digital.

En cuanto al modo de conservación, en la Filmoteca de Valencia, los DCP se almacenan desde el año 2010 con los discos duros y el vídeo, en una cámara a $18^{\circ}$ y $45-50 \%$ de humedad con renovación diaria de aire. En la Filmoteca de Catalunya, pensando ya en la más que probable desaparición del soporte físico, los soportes se almacenan en un servidor fiable, que cumple la normativa internacional TRAC, combinados con un software de trazabilidad con normativa OAIS. Tanto en el CGAI como en la Filmoteca de Andalucía el depósito se realiza en los propios almacenes de material fotoquímico, sin unas especiales condiciones de conservación, aunque en el caso de Andalucía se plantea como seguridad subirlos al servidor informático de la institución y además incorporarlo al propio disco duro que contiene el proyector digital DCP. En la Filmoteca Española, el criterio es la conservación dentro de un envase de plástico y en armario cerrado en almacén climatizado a $18^{\circ} \mathrm{C}$ y $40 \%$ de humedad.

De acuerdo con los datos genéricos de ámbito internacional aportados por la FIAF, y con las apreciaciones y casos prácticos más concretos recabados de las filmotecas nacionales analizadas, se pone de manifiesto que la instauración del formato DCP/ DCI en la industria cinematográfica contemporánea ha tenido un efecto constatable y real en la actividad filmotecaria. Una mayoría cualificada de los miembros de la FIAF han asimilado en el periodo 2010-2014 los estándares DCI como el material fílmico de referencia en el campo cinematográfico digital, adoptándolo, tanto en sus actividades de conservación y catalogación, como de programación y exhibición cinematográfica.

\section{DISCUSIÓN Y CONCLUSIONES}

La consolidación de la industria cinematográfica moderna se fundó sobre la creación de un estándar tecnológico diseñado por las grandes majors norteamericanas, dirigido a establecer un parámetro de control sobre los procesos de producción, distri- 
bución y exhibición fílmica. Este modelo, a través del estándar de $35 \mathrm{~mm}$. se ha mantenido vigente desde la primera década del siglo pasado hasta los albores del milenio.

La irrupción de la tecnología digital puso en duda las bases de este sistema productivo, al posibilitar la atomización de los procesos de producción, distribución y exhibición, lo que llevó a pensar en una desconcentración del mercado. La creación del sistema y nuevo estándar DCI en 2002, de homologación de parámetros técnicos de naturaleza digital, y de los formatos derivados de éste DSM, DCDM y DCP, fue la respuesta del grueso de la industria cinematográfica multinacional a esa posible dispersión.

El abandono de la producción de soportes fotoquímicos destinados a la industria cinematográfica en el cambio de siglo, así como la implantación acelerada a lo largo de la última década de la producción y exhibición cinematográfica industrial de naturaleza ya digital, han convertido al sistema DCI en la solución profesional hegemónica para la práctica totalidad de exhibidores, distribuidores y productores cinematográficos.

Las filmotecas, que en un principio mostraron reticencias a la introducción de los nuevos soportes digitales en el campo filmotecario, han adoptado de forma plena a lo largo del periodo 2010-2014 la tecnología digital, aceptando y convirtiendo mayoritariamente los formatos DCP/DCI y DCDM/DCI en equivalentes de obra cinematográfica original.

Una mayoría cualificada de los centros filmotecarios adscritos a la de la Federación Internacional de Archivos Fílmicos (FIAF) han asimilado en el periodo 2010-2014 los estándares DCI y los formatos industriales derivados de éste como el material fílmico de referencia en el campo cinematográfico digital, adoptándolo, tanto en sus actividades de almacenaje, conservación y catalogación, como de programación, exhibición y difusión cinematográfica.

El estudio de los casos prácticos de esta transición, aportados por la Filmoteca Española, la Filmoteca de Catalunya, la Filmoteca Valenciana, el CGAI y la Filmoteca de Andalucía, nos ha permitido constatar la exigencia común de compatibilidad DCP a los depositantes desde el año 2010, la disposición común de sistemas de reproducción DCP, el incremento constante, progresivo y mayoritario de copias DCP frente a cualquier otro formato de conservación fílmica en los últimos cinco años, así como los pasos en ciernes, en especial en la Filmoteca Española y en la Filmoteca de Catalunya, hacia la más que probable desaparición del soporte físico.

\section{REFERENCIAS}

BOE 2014. Boletín Oficial del Estado. Jueves 27 de febrero de 2014, 50, Sec. III. Pág. 18812-18829. Resolución de 20 de febrero, de la Dirección General del Instituto de la Cinematografía y de las Artes Audiovisuales, por la que se convocan para el año 2014 ayudas para la producción de largometrajes sobre proyecto.

BOJA 2013. Boletín Oficial de la Junta de Andalucía, 21 de agosto de 2013, 163, pp. 5-22. Orden de 8 de agosto, por la que se establecen las bases reguladoras para concesión de subvenciones, en régimen de concurrencia competitiva, de apoyo a empresas culturales y creativas andaluzas para el fomento de su competitividad, modernización e internacionalización.

BOJA 2011. Boletín Oficial de la Junta de Andalucía, 29 de agosto 2011, 169, pp. 5-68. Orden de 27 de julio, por la que se establecen las bases reguladoras de concesión de subvenciones en régimen de concurrencia competitiva al desarrollo de proyectos y a la producción de obras audiovisuales en Andalucía y se efectúa su convocatoria para 2011.

BOPV 2014. Boletín Oficial del País Vasco. 9 de Junio de 2014, 107, disp. 2506, pp. 1-20. Orden de 4 de junio de la Consejera de Educación, Política Lingüística y Cultura, por la que se convoca y regula la concesión de subvenciones en el ejercicio 2014 a la creación, desarrollo y producción audiovisual.

Borde, R. (1991). Los Archivos Cinematográficos. VaIencia; Ediciones IVAC- Filmoteca de Generalitat Valenciana.

Borde, R., y Buache, F. (1997). La crise des cinémathèques, et du monde. Paris; L'Age d'homme.

Bordwell, D. (1979). The art cinema as a mode of film practice. Film Criticism, 4(1), 56-64.

Cave, D. (2013). Digital Acquisition at the BFI. Journal of Film Preservation. April, 77-83.

Cherchi Usai, P (1995). La muerte del cine: historia y memoria cultural en el medioevo digital; prefacio de Martin Scorsese. Barcelona; Laertes-Filmoteca de Andalucía. 
Christopherson, S. (2008). Beyond the self expressive creative worker. An industry perspective on entertaiment media. Theory, Culture \& Society, vol. 25: 7-8, 89-112. http://dx.doi. org/10.1177/0263276408097797

Costa, J. M. (2004). Film Archives in Motion. Journal of Film Preservation, 68, 4-14.

Cubitt, S. (1998). Digital aesthetics. London; Sage.

Curtis, S. (2004). A house divided. The MPPC in Translation, en Keil, Ch; Stamp, S (editors). American Cinema's Transitional Era. Berkeley; University of California Press.

Darley, A. (2002). Visual digital culture: Surface play and spectacle in new media genres. Routledge.

Del Amo, A.; Fernández, J. (2011). Conservación audiovisual en el inicio de la era digital. San Sebastián; Filmoteca Española/Filmoteca Vasca.

FIAF Commissions (2010). Recommendation on the deposit and acquisition of D-cinema elements for long term preservation and access; http://www. fiafnet.org/uk/publications/fep.html [19/09/2014].

FIAF Commissions (2012). D-Cinema Equipment Frequently Asked Questions, by Torkell Sætervadet; http://www.fiafnet.org/uk/publications/fep.html [19/09/2014].

FIAF Annual Reports (2013). Brussels: FIAF Publications.

Fossati, G. (2009). From Grain to Pixel: The Archival Life of Film in Transition. Amsterdam; Amsterdam University Press. http://dx.doi. org/10.5117/9789089641397

Francis, D. (2002). Challenges of Film Archiving in the 21 st century. Journal of Film Preservation, 65, 18-24.

Fullerton, J.; Söderbergh-Widding, A. (2000). Moving images: from Edison to the webcam. Sidney; John Libbey \& Co Ltd.

García-Casado, P.; Alberich-Pascual, J. (2014). Filmotecas en la encrucijada. Función y expansión de la actividad filmotecaria en el nuevo escenario digital. El profesional de la información, 23(1), 5964. http://dx.doi.org/10.3145/epi.2014.ene.07

García-Santamaría, J. V. (2013). Digitalización de salas de cine en España: la oportunidad perdida de una potencia mundial en el sector de la exhibición. L'Atalante, Julio-Diciembre, 75-81.

Gubern, R. (1987). La mirada opulenta: explotación de la iconosfera contemporánea. Barcelona; Gustavo Gili.

Hanson, M. (2004). The End of Celluloid: Film futures in the digital age. RotoVision.

Hartley, J. (2008). From the Consciousness Industry to Creative Industries: Consumer-created content, social network markets and the growth of knowledge.
En J. Holt \& A. Perren. (Eds.). Media Industries: History, Theory and Methods. Oxford; Blackwell.

Izquierdo, J. (2007). Distribución y Exhibición cinematográficas en España. Un estudio de situación del negocio en la transición tecnológica digital. (Tesis doctoral). Castellón; Univ. Jaume I.

Jones, L. A. (1933). A historical summary of standardization in the Society of Motion Picture Engineers. Journal of the Society of Motion Picture Engineers, April, 1, 280-293. http://dx.doi. org/10.5594/j12977

López-Yepes, A. (2008). Filmotecas y archivos fílmicos en línea: producción, difusión, interconexión y posicionamiento en internet. Scire. 14: 2, 61.

Manley, B. (2011). Moving Pictures: The History of Early Cinema.

Manovich, L. (2001). The language of new media. Massachussets; MIT press.

Meyer, M. P. (2005). Traditional Film Proyection in a digital age. Journal of Film Preservation, 70, 15-19.

Mezias, J.; Boyle, E. (2005). Blind Trust: Market Control, Legal Environments, and the Dynamics of Competitive Intensity in the Early American Film Industry, 1893-1920. Administrative Science Quarterly, 50, 1-34.

Miller, T.; Stam, R. (Eds.). (1999). A companion to film theory. John Wiley \& Sons.

Musser, C. (1991). Before the Nickelodeon. Berkeley; University of California Press.

Nowak, A. (2010). Digital Cinema Technologies from the Archive's Perspective. AMIA Tech Review, 2.

Nowell-Smith, G. (Ed.). (1996). The Oxford history of world cinema. Oxford; Oxford University Press.

Paz-García, C. (2013). El agotamiento del modelo cinematográfico analógico: distribución y exhibición digital: un nuevo marco para la cooperación audiovisual iberoamericana. (Memoria para optar al grado de doctor). Madrid; Universidad Complutense de Madrid.

Schaefer, E.; Streible, D. (2002). Archival News (Film archives, access, acquisitions, preservation, institutions and organizations). Cinema Journal, 42:1, pp 122-129.

Sætervadet, T. (2006). The Advanced Projection Manual. Oslo; Norwegian Film Institute-FIAF.

Thorburn, D.; Jenkins, H. (Eds.). (2004). Rethinking media change: the aesthetics of transition. Massachussets; MIT Press.

Talens, J.; Zunzunegui, S. (Eds.). (1997). Historia general del cine: América (1915-1928).Vol. 4. Madrid; Anaya.

Tubella, I.; Tabernero, C.; Dwyer, V. (2008). Internet y televisión: la guerra de las pantallas. Barcelona; Ariel. 
Wengström, J. (2013). Collection Building and Programming in the Future. The fate of nonnational in archives in light of the change from $35 \mathrm{~mm}$ to DCP in theatrical distribution. Journal of Film Preservation. April, 17-20.
Wyver, J. (1989). Moving image: an international history of film, television and video. Basil Blackwell Ltd.

\section{ANEXO I}

\begin{tabular}{|c|c|c|c|c|}
\hline FILMOTECA / FILM ARCHIVE & Sede & $\begin{array}{l}\text { Exhibición } \\
\text { DCP }\end{array}$ & $\begin{array}{l}\text { Archivo } \\
\text { DCP }\end{array}$ & $\begin{array}{c}\text { Otros } \\
\text { sistemas } \\
\text { de archivo } \\
\text { digital }\end{array}$ \\
\hline CENTRO GALEGO DE ARTES DA IMAXE & A Coruña & $\mathrm{S}$ & $\mathrm{S}$ & $\mathrm{S}$ \\
\hline NATIONAL SCREEN AND SOUND ARCHIVE OF WALES & Aberystwyth & NC & NC & $\mathrm{S}$ \\
\hline LA CINEMATHEQUE ALGERIENNE & Alger & NC & NC & NC \\
\hline EYE FILM INSTITUTE NETHERLANDS & Amsterdam & $\mathrm{S}$ & $\mathrm{S}$ & $\bar{S}$ \\
\hline $\begin{array}{l}\text { TAINIOTHIKI TIS ELLADOS } \\
\text { GREEK FILM ARCHIVE }\end{array}$ & Athens & NC & NC & S \\
\hline FILM ARCHIVE & Bangkok & NC & $\mathrm{S}$ & $\mathrm{S}$ \\
\hline FILMOTECA DE CATALUNYA & Barcelona & $\mathrm{S}$ & $\mathrm{S}$ & $\mathrm{S}$ \\
\hline CHINA FILM ARCHIVE & Beijing & $\mathrm{S}$ & $\mathrm{S}$ & $\mathrm{S}$ \\
\hline NATIONAL CINEMATEQUE OF LEBANON & Beirut & NC & NC & $\mathrm{S}$ \\
\hline JUGOSLOVENSKA KINOTEKA & Beograd & NC & NC & $\mathrm{S}$ \\
\hline PACIFIC FILM ARCHIVE University of California & Berkeley & $\mathrm{S}$ & $\mathrm{S}$ & $\mathrm{S}$ \\
\hline BUNDESARCHIV-FILMARCHIV & Berlin & NC & NC & $\bar{S}$ \\
\hline DEUTSCHE KINEMATHEK & Berlin & $\mathrm{S}$ & $\mathrm{S}$ & $\mathrm{S}$ \\
\hline LICHTSPIEL / KINEMATHEK BERN & Berne & $\mathrm{S}$ & $\mathrm{S}$ & $\mathrm{S}$ \\
\hline INDIANA UNIVERSITY LIBRARIES FILM ARCHIVE & Bloomington & $\mathrm{S}$ & $\mathrm{S}$ & $\mathrm{S}$ \\
\hline FUNDACION PATRIMONIO FILMICO COLOMBIANO & Bogotá & $\mathrm{S}$ & $\mathrm{S}$ & $\mathrm{S}$ \\
\hline CINETECA DEL COMUNE DI BOLOGNA & Bologna & $\mathrm{S}$ & $\mathrm{S}$ & $\mathrm{S}$ \\
\hline SLOVAK FILM INSTITUTE & Bratislava & $\mathrm{S}$ & $\mathrm{S}$ & $\mathrm{S}$ \\
\hline $\begin{array}{l}\text { AUSTRALIAN CINEMATHEQUE, QUEENSLAND ART GAL- } \\
\text { LERY / GALLERY OF MODERN ART }\end{array}$ & Brisbane & S & S & S \\
\hline $\begin{array}{l}\text { CINEMATHEQUE ROYALE DE BELGIQUE / KONINKLIJK } \\
\text { BELGISCH FILMARCHIEF }\end{array}$ & Bruxelles & S & S & S \\
\hline ARHIVA NATIONALA DE FILME - CINEMATECA ROMANA & Bucuresti & $\mathrm{S}$ & $\mathrm{S}$ & $\mathrm{S}$ \\
\hline HUNGARIAN DIGITAL FILM ARCHIVE & Budapest & $\mathrm{S}$ & $\mathrm{S}$ & $\mathrm{S}$ \\
\hline FUNDACION CINEMATECA ARGENTINA & Buenos Aires & $\mathrm{S}$ & $\mathrm{S}$ & $\mathrm{S}$ \\
\hline DANISH FILM INSTITUTE & Coopenhague & $\mathrm{S}$ & $\mathrm{S}$ & $\mathrm{S}$ \\
\hline FILMOTECA DE ANDALUCIA & Cordoba & $\mathrm{S}$ & $\mathrm{S}$ & $\mathrm{S}$ \\
\hline BANGLADESH FILM ARCHIVE & Dhaka & $\mathrm{N}$ & $\mathrm{N}$ & $\mathrm{N}$ \\
\hline IRISH FILM ARCHIVE & Dublin & $\mathrm{N}$ & $\mathrm{S}$ & $\mathrm{S}$ \\
\hline FILMMUSEUM LANDESHAUPTSTADT & Düsseldorf & $\mathrm{S}$ & $\mathrm{S}$ & $\mathrm{S}$ \\
\hline MEDIATECA REGIONALE TOSCANA FILM COMMISSION & Firenze & $\mathrm{S}$ & $\mathrm{S}$ & $\mathrm{S}$ \\
\hline DEUTSCHES FILMINSTITUT DIF & Frankfurt & $\mathrm{S}$ & $\mathrm{S}$ & $\mathrm{S}$ \\
\hline DEUTSCHES FILMINSTITUT DIF (Film Archive) & Frankfurt & $\mathrm{S}$ & $\mathrm{S}$ & $\mathrm{S}$ \\
\hline FUKUOKA CITY PUBLIC LIBRARY FILM ARCHIVE & Fukuoka & $\mathrm{S}$ & $\mathrm{S}$ & $\mathrm{S}$ \\
\hline CINETECA DEL FRIULI & Gemona & $\mathrm{N}$ & $\mathrm{S}$ & $\mathrm{S}$ \\
\hline
\end{tabular}




\begin{tabular}{|c|c|c|c|c|}
\hline FILMOTECA / FILM ARCHIVE & Sede & $\begin{array}{l}\text { Exhibición } \\
\text { DCP }\end{array}$ & $\begin{array}{l}\text { Archivo } \\
\text { DCP }\end{array}$ & $\begin{array}{c}\text { Otros } \\
\text { sistemas } \\
\text { de archivo } \\
\text { digital }\end{array}$ \\
\hline SCOTTISH SCREEN ARCHIVE & Glasgow & $\mathrm{N}$ & $\mathrm{S}$ & $\mathrm{S}$ \\
\hline CINEMATHEQUE DE GRENOBLE & Grenoble & $\mathrm{S}$ & $\mathrm{S}$ & $\mathrm{S}$ \\
\hline VIETNAM FILM ARCHIVE & Hanói & NC & $\mathrm{N}$ & $\mathrm{N}$ \\
\hline NATIONAL AUDIOVISUAL ARCHIVE & Helsinki & $\mathrm{S}$ & $\mathrm{S}$ & $\mathrm{S}$ \\
\hline HONG KONG FILM ARCHIVE 3/F Resource Centre & Hong Kong & $\mathrm{N}$ & $\mathrm{N}$ & $\mathrm{N}$ \\
\hline TURKISH FILM AND TV INSTITUTE & Istambul & $\mathrm{S}$ & $\mathrm{S}$ & $\mathrm{S}$ \\
\hline ECPAD & Ivry & $\mathrm{N}$ & $\mathrm{N}$ & $\mathrm{N}$ \\
\hline ISRAEL FILM ARCHIVE/JERUSALEM CINEMATHEQUE & Jerusalem & $\mathrm{S}$ & $\mathrm{S}$ & $\mathrm{S}$ \\
\hline ARCHIVE ASSOCIATES PTY LTD & Kambah & $\mathrm{N}$ & $\mathrm{N}$ & $\mathrm{N}$ \\
\hline SILESIAN FILM ARCHIV / CENTER OF FILM ART & Katowice & $\mathrm{S}$ & $\mathrm{S}$ & $\mathrm{S}$ \\
\hline UKRAINIAN STATE FILM AGENCY & Kiev & $\mathrm{N}$ & $\mathrm{N}$ & $\mathrm{S}$ \\
\hline CINEMATHEQUE SUISSE & Lausanne & $\mathrm{S}$ & $\mathrm{S}$ & $\mathrm{S}$ \\
\hline FILMOTECA UNIVERSIDAD CATOLICA & Lima & $\mathrm{N}$ & $\mathrm{N}$ & $\mathrm{s}$ \\
\hline CINEMATECA PORTUGUESA MUSEU DO CINEMA & Lisboa & $\mathrm{S}$ & $\mathrm{S}$ & $\mathrm{S}$ \\
\hline $\begin{array}{l}\text { ARHIV REPUBLIKE SLOVENIJE / SLOVENSKI FILMSKI } \\
\text { ARHIV }\end{array}$ & Ljubljana & $\mathrm{N}$ & $\mathrm{N}$ & $\mathrm{N}$ \\
\hline SLOVENIAN CINEMATHEQUE / SLOVENSKA KINOTEKA & Ljubljana & $\mathrm{S}$ & $\mathrm{S}$ & $\mathrm{S}$ \\
\hline BRITISH FILM INSTITUTE National Archive & London & $\mathrm{S}$ & $\mathrm{S}$ & $\mathrm{S}$ \\
\hline FILM ARCHIVE / IMPERIAL WAR MUSEUMS & London & $\mathrm{s}$ & $\mathrm{S}$ & $\mathrm{S}$ \\
\hline $\begin{array}{l}\text { ACADEMY FILM ARCHIVE - Center for Motion Picture } \\
\text { Study }\end{array}$ & Los Angeles & $\mathrm{S}$ & S & $\mathrm{S}$ \\
\hline UCLA FILM \& TELEVISION ARCHIVE & Los Angeles & $\mathrm{S}$ & $\mathrm{S}$ & $\mathrm{S}$ \\
\hline $\begin{array}{l}\text { AMERICAN FILM INSTITUTE } \\
\text { Los Angeles Campus }\end{array}$ & $\begin{array}{l}\text { Los Angeles - } \\
\text { Washington }\end{array}$ & $\mathrm{S}$ & $\mathrm{S}$ & $\mathrm{S}$ \\
\hline AMERICAN FILM INSTITUTE Collection & $\begin{array}{l}\text { Los Angeles - } \\
\text { Washington }\end{array}$ & $\mathrm{S}$ & $\mathrm{S}$ & $\mathrm{s}$ \\
\hline CENTRE NATIONAL DE L'AUDIOVISUEL & Luxembourg & $\mathrm{S}$ & $\mathrm{S}$ & $\mathrm{S}$ \\
\hline CINEMATHEQUE DE LA VILLE DE LUXEMBOURG & Luxembourg & $\mathrm{S}$ & $\mathrm{S}$ & $\mathrm{s}$ \\
\hline INSTITUT LUMIERE & Lyon & $\mathrm{s}$ & $\mathrm{S}$ & $\mathrm{s}$ \\
\hline FILMOTECA ESPAÑOLA & Madrid & $\mathrm{S}$ & $\mathrm{S}$ & $\mathrm{S}$ \\
\hline NORTH WEST FILM ARCHIVE & Manchester & $\mathrm{N}$ & $\mathrm{N}$ & $\mathrm{S}$ \\
\hline AUSTRALIAN CENTRE FOR THE MOVING IMAGE - ACMI & Melbourne & $\mathrm{S}$ & $\mathrm{S}$ & $\mathrm{S}$ \\
\hline CENTRO DE CAPACITACION CINEMATOGRAFICA - CCC & Mexico & $\mathrm{S}$ & $\mathrm{S}$ & $\mathrm{S}$ \\
\hline CINETECA NACIONAL & Mexico & $\mathrm{S}$ & $\mathrm{S}$ & $\mathrm{S}$ \\
\hline FILMOTECA DE LA UNAM & Mexico & $\mathrm{S}$ & $\mathrm{S}$ & $\mathrm{s}$ \\
\hline FONDAZIONE CINETECA ITALIANA & Milano & $\mathrm{S}$ & $\mathrm{S}$ & $\mathrm{s}$ \\
\hline $\begin{array}{l}\text { CINETECA NUEVO LEON - CENTRO DE LAS ARTES CON- } \\
\text { SEJO PARA LA CULTURA Y LAS ARTES }\end{array}$ & Monterrey & N & S & $\mathrm{S}$ \\
\hline ARCHIVO NACIONAL DE LA IMAGEN & Montevideo & $\mathrm{N}$ & $\mathrm{N}$ & $\mathrm{N}$ \\
\hline CINEMATECA URUGUAYA & Montevideo & $\mathrm{N}$ & $\mathrm{N}$ & $\mathrm{N}$ \\
\hline LA CINEMATHEQUE QUEBECOISE & Montreal & $\mathrm{S}$ & $\mathrm{S}$ & $\mathrm{S}$ \\
\hline GOSFILMOFOND OF RUSSIA & Moscú & $\mathrm{S}$ & $\mathrm{S}$ & $\mathrm{S}$ \\
\hline FILMMUSEUM IM MÜNCHNER STADTMUSEUM & Muenchen & $\mathrm{S}$ & $\mathrm{S}$ & $\mathrm{S}$ \\
\hline ANTHOLOGY FILM ARCHIVES & New York & S & S & S \\
\hline
\end{tabular}




\begin{tabular}{|c|c|c|c|c|}
\hline FILMOTECA / FILM ARCHIVE & Sede & $\begin{array}{l}\text { Exhibición } \\
\text { DCP }\end{array}$ & $\begin{array}{l}\text { Archivo } \\
\text { DCP }\end{array}$ & $\begin{array}{c}\text { Otros } \\
\text { sistemas } \\
\text { de archivo } \\
\text { digital }\end{array}$ \\
\hline DEPARTMENT OF FILM - THE MUSEUM OF MODERN ART & New York & $\mathrm{S}$ & $\mathrm{S}$ & $\mathrm{s}$ \\
\hline CINEMATHEQUE DE NICE & Nice & $\mathrm{S}$ & $\mathrm{S}$ & $\mathrm{s}$ \\
\hline NORWEGIAN FILM INSTITUTE & Oslo & $\mathrm{S}$ & $\mathrm{S}$ & $\mathrm{S}$ \\
\hline $\begin{array}{l}\text { BIBLIOTHEQUE NATIONALE DE FRANCE DEPT. DE } \\
\text { L'AUDIOVISUEL }\end{array}$ & Paris & $\mathrm{S}$ & S & $\mathrm{S}$ \\
\hline CINEMATHEQUE FRANCAISE / MUSEE DU CINEMA & Paris & $\mathrm{S}$ & $\mathrm{S}$ & $\mathrm{S}$ \\
\hline FORUM DES IMAGES & Paris & $\mathrm{S}$ & $\mathrm{S}$ & $\mathrm{s}$ \\
\hline $\begin{array}{l}\text { ICONOTHEQUE DE L'INSTITUT NATIONAL DU SPORT, } \\
\text { DE L'EXPERTISE ET DE LA PERFORMANCE }\end{array}$ & Paris & $\mathrm{S}$ & S & $\mathrm{S}$ \\
\hline CENTRE NATIONAL DU CINEMA ET DE L'IMAGE ANIMEE & $\begin{array}{l}\text { Paris - Bois } \\
\text { d'Arcy }\end{array}$ & $\mathrm{S}$ & $\mathrm{S}$ & $\mathrm{S}$ \\
\hline INSTITUT JEAN VIGO Cinémathèque Euro-régionale & Perpignan & $\mathrm{N}$ & $\mathrm{N}$ & $\mathrm{N}$ \\
\hline LA CORSE ET LE CINEMA CINEMATHEQUE DE CORSE & Porto Vecchio & $\mathrm{N}$ & $\mathrm{N}$ & $\mathrm{N}$ \\
\hline NARODNI FILMOVY ARCHIV & Praha & $\mathrm{s}$ & $\mathrm{S}$ & $\mathrm{s}$ \\
\hline $\begin{array}{l}\text { SOUTH AFRICAN NATIONAL FILM, VIDEO AND SOUND } \\
\text { ARCHIVES }\end{array}$ & Pretoria & $\mathrm{N}$ & $\mathrm{N}$ & $\mathrm{N}$ \\
\hline NATIONAL FILM ARCHIVE OF INDIA & Pune & $\mathrm{N}$ & $\mathrm{N}$ & $\mathrm{N}$ \\
\hline CINEMATHEQUE MAROCAINE / CCM & Rabat & $\mathrm{N}$ & $\mathrm{N}$ & $\mathrm{N}$ \\
\hline KVIKMYNDASAFN ISLANDS & Reykjavik & $\mathrm{N}$ & $\mathrm{N}$ & $\mathrm{S}$ \\
\hline CINEMATECA DO MUSEU DE ARTE MODERNA & Rio de Janeiro & $\mathrm{N}$ & $\mathrm{N}$ & $\mathrm{N}$ \\
\hline GEORGE EASTMAN HOUSE & Rochester & $\mathrm{S}$ & $\mathrm{S}$ & $\mathrm{S}$ \\
\hline $\begin{array}{l}\text { FONDAZIONE CENTRO SPERIMENTALE DI CINEMATO- } \\
\text { GRAFIA / CINETECA NAZIONALE }\end{array}$ & Roma & $\mathrm{S}$ & S & $\mathrm{S}$ \\
\hline $\begin{array}{l}\text { EUSKADIKO FILMATEGIA FUNDAZIOA- FUNDACIÓN } \\
\text { FILMOTECA VASCA }\end{array}$ & San Sebastian & $\mathrm{N}$ & S & $\mathrm{S}$ \\
\hline CINETECA NACIONAL DE CHILE & Santiago & $\mathrm{S}$ & $\mathrm{S}$ & $\mathrm{S}$ \\
\hline CINEMATECA DOMINICANA & Santo Domingo & $\mathrm{N}$ & $\mathrm{N}$ & $\mathrm{N}$ \\
\hline CINEMATECA BRASILEIRA & Sao Paulo & $\mathrm{S}$ & $\mathrm{S}$ & $\mathrm{s}$ \\
\hline KINOTEKA BOSNE I HERCEGOVINE & Sarajevo & $\mathrm{N}$ & $\mathrm{N}$ & $\mathrm{s}$ \\
\hline KOREAN FILM ARCHIVE & Seoul & $\mathrm{s}$ & $\mathrm{S}$ & $\mathrm{s}$ \\
\hline ASIAN FILM ARCHIVE & Singapore & $\mathrm{N}$ & $\mathrm{N}$ & $\mathrm{S}$ \\
\hline KINOTEKA NA MAKEDONIJA & Skopje & $\mathrm{s}$ & $\mathrm{S}$ & $\mathrm{s}$ \\
\hline BULGARSKA NACIONALNA FILMOTEKA & Sofia & $\mathrm{S}$ & $\mathrm{S}$ & $\mathrm{S}$ \\
\hline SVENSKA FILMINSTITUTET & Stockholm & $\mathrm{S}$ & $\mathrm{S}$ & $\mathrm{S}$ \\
\hline CHINESE TAIPEI FILM ARCHIVE & Taipei & $\mathrm{N}$ & $\mathrm{N}$ & $\mathrm{N}$ \\
\hline ESTONIAN FILM ARCHIVES & Tallinn & $\mathrm{N}$ & $\mathrm{N}$ & $\mathrm{s}$ \\
\hline CINEMATHEQUE DE TANGER & Tanger & $\mathrm{N}$ & $\mathrm{N}$ & $\mathrm{N}$ \\
\hline GEORGIAN NATIONAL FILM CENTER & Tbilisi & $\mathrm{N}$ & $\mathrm{N}$ & $\mathrm{S}$ \\
\hline THESSALONIKI CINEMA MUSEUM & Thessalonique & $\mathrm{N}$ & $\mathrm{N}$ & $\mathrm{N}$ \\
\hline ARKIVI QENDROR SHTETErORI FILMIT & Tirana & $\mathrm{N}$ & $\mathrm{N}$ & $\mathrm{S}$ \\
\hline NATIONAL FILM CENTER \& NATIONAL MUSEUM & Tokyo & $\mathrm{S}$ & $\mathrm{S}$ & $\mathrm{S}$ \\
\hline $\begin{array}{l}\text { MUSEO NAZIONALE DEL CINEMA FONDAZIONE M. } \\
\text { ADRIANA PROLO }\end{array}$ & Torino & $\mathrm{S}$ & S & $\mathrm{S}$ \\
\hline FILM REFERENCE LIBRARY / TIFF CINEMATHEQUE & Toronto & $\mathrm{S}$ & $\mathrm{S}$ & $\mathrm{S}$ \\
\hline CINEMATHEQUE DE TOULOUSE & Toulouse & $\mathrm{S}$ & $\mathrm{S}$ & $\mathrm{S}$ \\
\hline
\end{tabular}




\begin{tabular}{|c|c|c|c|c|}
\hline FILMOTECA / FILM ARCHIVE & Sede & $\begin{array}{l}\text { Exhibición } \\
\text { DCP }\end{array}$ & $\begin{array}{l}\text { Archivo } \\
\text { DCP }\end{array}$ & $\begin{array}{c}\text { Otros } \\
\text { sistemas } \\
\text { de archivo } \\
\text { digital }\end{array}$ \\
\hline $\begin{array}{l}\text { INSTITUTO VALENCIANO DE L'AUDIOVISUAL RICARDO } \\
\text { MUÑOZ SUAY }\end{array}$ & Valencia & $\mathrm{S}$ & S & $\mathrm{S}$ \\
\hline FILMOTEKA NARODOWA & Warszawa & $\mathrm{S}$ & $\mathrm{S}$ & $\mathrm{S}$ \\
\hline $\begin{array}{l}\text { MOTION PICTURE, BROADCAST AND RECORDED } \\
\text { SOUND DIVISION }\end{array}$ & Washington & $\mathrm{S}$ & $\mathrm{S}$ & S \\
\hline NATIONAL GALLERY OF ART - FILM DEPARTMENT & Washington & $\mathrm{S}$ & $\mathrm{S}$ & $\mathrm{S}$ \\
\hline THE NEW ZEALAND FILM ARCHIVE & Wellington & $\mathrm{S}$ & $\mathrm{S}$ & NC \\
\hline FILMARCHIV AUSTRIA & Wien & $\mathrm{S}$ & $\mathrm{S}$ & $\mathrm{S}$ \\
\hline OESTERREICHISCHES FILMMUSEUM & Wien & $\mathrm{S}$ & $\mathrm{S}$ & $\mathrm{S}$ \\
\hline
\end{tabular}

Fuente: Anuario FIAF 2013; elaboración propia. 